\title{
Le Français sur Objectifs universitaires pour les professeurs des écoles
}

\author{
Ana Vujović
}

Faculté de formation des maîtres, Université de Belgrade*

Il est évident que pour les étudiants de différentes facultés non-philologiques la connaissance d'une langue étrangère comprend, avant tout, les compétences linguistiques dans le domaine de leur future profession (ce qu'on appelle, le plus souvent, une langue sur objectifs spécifiques ou une langue de spécialité). En plus, ces dernières années, l'internationalisation de l'enseignement supérieur et l'expansion de la mobilité étudiante influencent fortement le fonctionnement des universités européennes. Grâce aux programmes de coopération développés entre la Faculté de formation des maîtres de l'Université de Belgrade et les ESPE de Bordeaux et de Lyon, nos étudiants peuvent intégrer les programmes de mobilité universitaire Erasmus+ (au niveau de Master I et II), ainsi qu'exercer une partie de leur stage professionnel dans un milieu francophone. Vues ces nouvelles possibilités de découvrir un milieu académique avec une organisation, des contenus et des méthodes de travail très différents de ceux qu'ils ont connus dans leurs pays d'origine, l'objectif de notre recherche est de détecter les besoins concrets de nos étudiants (ceux qui désirent intégrer un programme de mobilité dans l'espace francophone, ceux qui envisagent de poser leur candidature pour une bourse en vue d'un master en France ou dans un pays francophone et ceux qui désirent partir pour un stage professionnel dans une ESPE). À partir d'une analyse détaillée de ces besoins concrets, en utilisant la méthode descriptive et comparative, nous proposerons des contenus possibles du programme du FOU pour les professeurs des écoles, ainsi que les supports, les compétences travaillées et les activités.

Mots-clés : FOU, programme, compétences travaillées, supports, activités, professeurs des écoles

\section{Introduction}

En mars 2018, à la Faculté de formation des maitres de 1'Université de Belgrade a eu lieu une formation consacrée au français sur objectif spécifique

\footnotetext{
" ana.vujovic@uf.bg.ac.rs
} 
et au français sur objectif universitaire. Organisée par la Section des professeurs universitaires des langues sur objectif spécifique et soutenue par l'Agence universitaire de la francophonie et l'Institut français de Belgrade, la formation a été assurée par Chantal Parpette et s'est déroulée en deux temps : 2 jours ont été consacrés au FOS et 3 jours au FOU. Il s'agissait de mener une réflexion autour des enjeux, et de la construction de programmes de FOS dans le champ professionnel et universitaire en relation avec les spécialités étudiées dans les départements de l'université. La formation a également porté sur la didactisation des supports de cours, en particulier en compréhension de l'oral et de l'écrit. Il s'agissait à la fois de conforter les notions structurant le FOS-FOU (étapes de la démarche, rôles combinés d'information culturelle et de formation linguistique, types de données-supports, relation entre didactique et institution, etc.) et de développer des savoir-faire en programmation et didactisation. Les séances se sont donc essentiellement déroulées sous forme de travaux de groupe (études de cas, constructions de programmes, élaboration de consignes, et analyses critiques) entrecoupés de courtes présentations magistrales.

La décision qui a été prise de poursuivre le travail et de lui donner une visibilité par la publication de fiches pédagogiques sur différents sites internet est tout à fait positive et susceptible de regrouper un plus grand nombre d'enseignants autour de la question de l'enseignement du français en Serbie. Donc, la dissémination des résultats de cette formation auprès des enseignants (lors de différents séminaires et colloques) est un de ses objectifs et une tâche importante pour tous les participants.

\section{Distinction des notions-clés}

La distinction créée au sein du FLE par la notion de FOS a conduit à la création de celle de français général désignant toute la partie du FLE qui n'est pas du FOS. Le français général (FG) a un objectif large avec une grande diversité de thèmes et de compétences visées ; l'enseignant maitrise bien tous les contenus et peut travailler en autonomie, dispose d'un matériel déjà existant et même d'un grand choix de manuels. Par contre, le français sur objectif spécifique a un objet précis centré sur certaines situations et compétences cibles ; les contenus sont souvent nouveaux pour l'enseignant qui est obligé de prendre contact avec les acteurs du milieu étudié et d'élaborer le matériel pédagogique. D'habitude, la formation pour le français général est à moyen ou long terme, tandis que celle du FOS se fait souvent à court terme, même en urgence. Mais l'aspect sur lequel les deux démarches se rejoignent presque totalement est celui des activités didactiques : le FOS n'a pas inventé d'activités d'enseignement particulières. (Mangiante, Parpette, 2004 : 153-159) Le FOS n'est pas un domaine séparé du 
FLE mais un sous-champ du FLE et le meilleur moyen de comprendre ce qu'est la méthodologie communicative.

Les notions connexes mais distinctes sont :

- Français sur objectif spécifique qui est lié à une demande / un besoin clairement identifié(e), besoin extérieur à l'apprentissage de la langue

- Français de spécialité qui est lié à un domaine professionnel mais sans besoin clairement identifié (concernant les situations de communication ou les compétences)

- Français thématique qui ne dépend pas des besoins extérieurs particuliers et qui est une sorte du français général centré sur un thème spécifique (qui n'appartient pas nécessairement à un domaine professionnel).

Dans le cadre du FOS on distingue la notion du Français sur Objectif universitaire (FOU) qui comprend, au-delà de la compétence linguistique, une compétence de communication dans le cadre universitaire qui est très influencée par la dimension culturelle. La préoccupation interculturelle est aussi présente en FOS dans la mesure où la plupart des programmes visent à intégrer les apprenants dans un environnement professionnel français ou francophone déterminé (informations socio-économiques et politiques, habitudes de travail et relations professionnelles), mais elle est davantage présente et évidente dans le domaine du FOU.

Le contexte du FOU aussi peut être différent pour les étudiants qui étudient en français dans leur pays d'origine et pour ceux qui vont en mobilité dans un pays francophone, car l'acculturation au milieu universitaire français ou autre crée des situations de communication et exige des compétences différentes par rapport à celles d'une filière francophone dans le pays d'origine. Il peut être approché et travaillé au sein d'une discipline professionnelle répondant aux besoins spécifiques (par exemple, le FOU pour les étudiants en médecine qui partent en stage dans un hôpital français ou pour les futurs professeurs des écoles qui partent en master à une ESPE), mais aussi hors d'une discipline particulière envisageant des besoins communs à tous (une formation en FOU pour tous les boursiers serbes partant en mobilité en France, par exemple). D'où proviennent des stratégies transversales pour un public diversifié ou des stratégies disciplinaires pour un public de la même discipline.

\section{Le programme d'introduction au FOU pour les $P E$}

L'internationalisation de l'enseignement supérieur et l'expansion de la mobilité étudiante ont modifié le paysage universitaire en Europe et ailleurs. Chaque année, différents programmes d'échanges et mobilités universitaires, ainsi que de nombreuses bourses sont offerts à nos étudiants. Pour eux, c'est l'occasion de découvrir un milieu académique avec une organisation, de contenus et méthodes 
de travail différents de ceux qu'ils ont connus dans leur pays d'origine. La Faculté de formation des maîtres de l'Université de Belgrade développe la coopération avec plusieurs ESPE en France (celles de Lyon et Bordeaux), mais nos étudiants - futurs professeurs des écoles (PE), n'ont pas la possibilité de faire des études en français chez eux, dans une filière délocalisée d'une université française partenaire. Ce qu'ils ont, néanmoins, c'est la possibilité (s'ils sont intéressés et si leurs compétences langagières le permettent) de passer en France un semestre d'études dans le cadre d'Erasmus ${ }^{+}$, de faire un stage de trois semaines dans une école élémentaire française ou encore de poursuivre leurs études supérieures avec une bourse du gouvernement français.

Avec la baisse sensible du niveau linguistique de nos étudiants, les cours de langue française leur sont indispensables pour réussir leurs études ou le stage en français. En préparant ce programme du FOU, nous avons voulu aider nos étudiants - futurs professeurs des écoles - à se préparer au mieux sur le plan langagier afin que leur expérience professionnelle soit optimale. Notre objectif n'est pas de leur faire apprendre leur future profession (c'est-à-dire d'étudier ce qu'ils étudient déjà dans leur langue maternelle) ni de leur permettre d'exercer vraiment en langue française (puisqu'ils ne travailleront pas dans une école française ou francophone), mais de leur permettre de se débrouiller dans un milieu professionnelle francophone (soit universitaire, s'ils partent en mobilité, soit scolaire, s'ils sont en stage pratique). Nos étudiants rencontrent des difficultés à suivre un cursus en français, à prendre des notes tout particulièrement, à restituer des notions théoriques dans un plan cohérent ou à réaliser un rapport de stage. Nous avons voulu leur fournir des connaissances de base du français universitaire et répondre à quelques questions qu'ils pourraient se poser dans les différentes étapes de leurs études. Les contenus prévus comprennent avant tout la présentation du fonctionnement de l'enseignement supérieur français et ensuite leur préparation à étudier dans un nouvel environnement culturel et à s'approprier la méthodologie des activités universitaires nécessaires à la réussite académique.

Écouter un enseignant n'a jamais un impact aussi fort que d'aller soi-même chercher une information, la trouver, la classer, la comparer avec d'autres et finalement en faire une synthèse. Recentrer le contenu sur les étudiants et leurs besoins concrets implique aussi une prise en compte de leur rythme d'apprentissage sans oublier les apports en termes d'autonomie et de flexibilité. Afin de permettre aux étudiants de se rapprocher du milieu universitaire et professionnel, on propose des documents authentiques : divers syllabus de Licence et de Master, cours magistraux, entretiens avec des enseignants, témoignages des étudiants et des professeurs des écoles, lettres de motivation, rapports de stage, etc.

Il est conseillé et prévu de commencer ce programme avec un niveau A2, à l'écrit comme à l'oral. Le niveau de français visé à l'issue de ce travail est B1 pour l'ensemble des compétences. Organisés en 10 unités et 40 heures de 
travail, on propose des contenus concernant : l'organisation des ESPE ; les documents et démarches nécessaires à la candidature pour une mobilité ou pour une bourse ; renseignements sur l'inscription, l'arrivée au campus, le logement, la restauration, le fonctionnement des CDI ; compréhension des discours universitaires (cours magistraux, travaux dirigés) ; informations essentielles sur les structures possibles des travaux écrits à l'université, l'évaluation, l'organisation des examens et le comportement par rapport aux professeurs/étudiants/employés de service ; résolution des problèmes ; vie étudiante et activités extrascolaires (dans le campus ou en ville).

Pour pouvoir travailler chacune des quatre compétences linguistiques fondamentales avec lesquelles les étudiants sont déjà familiers (compréhension et production orale, compréhension et production écrite) mais prenant en compte les besoins des différents groupes d'étudiants, nous envisageons plusieurs supports : site des Académies, des universités et des ESPE, ainsi que de l'Institut français et de l'AUF ; programmes d'études ; documents et formulaires différents (Candidature pour les étudiants entrants, Contrat de mobilité, Visa de long séjour, Fiche de situation familiale, Fiche d'information, Appel à candidatures pour les bourses de mobilité d'études, assurance maladie, réclamations etc.); exemples de $\mathrm{CV}$ et de lettres de motivation comme modèle de rédaction; plusieurs séquences du site Étudier en francophonie ; règlements des universités et des résidences universitaires ; vidéos des cours magistraux et des travaux dirigés ; travaux écrits des étudiants ; vidéos, photos, brochures ou sites internet des bibliothèques ; manuels utilisés dans l'enseignement à l'ESPE; fiches d'évaluation et de notation ; exemples des examens des sessions précédentes ; témoignages des étudiants serbes rentrant de France ou des stagiaires français venant en Serbie ; photos et vidéos des activités sportives, culturelles et artistiques.

Les unités commencent le plus souvent par un exercice de compréhension orale (à partir d'un vidéo) ou écrite (à partir d'un site officiel) et on vérifie ce que les étudiants ont compris (d'abord dans l'ensemble et ensuite dans les détails du document). La grammaire est étudiée en fonction des situations dans lesquelles elle apparait.

Nous envisageons de commencer par des activités simples, comme : trouver les informations sur les sites officiels, lire l'emploi du temps et demander des précisions/explications, trouver le lieu de stage, se présenter dans un milieu inconnu (accueil de l'ESPE ou du campus universitaire, école élémentaire etc.), visionner un document vidéo présentant l'institution d'accueil. On continue par comprendre et remplir les documents nécessaires, rédiger son $\mathrm{CV}$ et la lettre de motivation, téléphoner ou écrire un courriel pour se renseigner, demander des renseignements oralement ou sous forme écrite, simuler des entretiens dans la rue, dans le campus et à l'université. Le vocabulaire est abordé en fonction des thématiques et de nombreux liens vers les pages web sont proposés. On travaille 
les éléments essentiels du vocabulaire administratif et judiciaire (pour pouvoir remplir les formulaires et comprendre les informations officielles). On propose d'analyser les manuels, les situations professionnelles et les travaux écrits des étudiants, de faire le plan d'un mémoire, de faire les commentaires des enregistrements et comparer avec la situation dans sa propre faculté.

Dans un programme d'apprentissage du FOU, les sujets culturels ne sont pas donnés à titre d'information complémentaire (ce qui est souvent le cas pour le FOS) mais sont travaillés sous la forme de diverses activités. Il faudrait toujours envisager plusieurs activités appliquées à la tâche professionnelle se terminant par un exercice de production orale. Cet exercice est particulièrement important : il a pour objectif d'aider les étudiants à faire la synthèse des compétences étudiées et de les mettre en situation. Il constitue un résumé de ce dont ils ont besoin pour leur stage professionnel ou pour leurs études en France.

Une fois le programme d'enseignement élaboré, nous constituons un corpus de supports authentiques sélectionnés en milieu universitaire mais aussi scolaire. Il suit une analyse pré-pédagogique et l'élaboration des activités didactiques.

On envisage d'utiliser des données déjà existantes dans les différents manuels du FOU (français et autres) ou sur les sites officiels des institutions universitaires et scolaires, ou encore des sites comme Étudier en Francophonie qui présente des situations réelles (cours, exposés, échanges administratifs ou de la vie quotidienne des étudiants etc.). On pense aussi à la préparation de données sollicitées particulièrement pour les besoins concrets de nos étudiants : interviews avec nos étudiants ayant déjà participé à une mobilité universitaire ou exercé un stage pratique en France, mais aussi avec les stagiaires français qui viennent en stage en Serbie et qui peuvent témoigner de différents aspects de la vie étudiante en France.

Le corpus répond aux besoins d'apprentissage de nos étudiants, mais il ne s'agit pas de collecter tout et n'importe quel texte moissonné au hasard sur internet. La représentativité du corpus est fondamentale pour intégrer la question de l'identité professionnelle dans la conception d'une formation qui s'appuie ainsi sur les données textuelles authentiques représentant des discours professionnels et universitaires que les apprenants sont amenés à maîtriser. L'enseignant, dans une phase préliminaire, se sert du corpus pour identifier et analyser des faits linguistiques et discursifs propres au discours étudié et qui pourraient être didactisés ultérieurement. Il s'en sert également pour en tirer des exemples qui vont enrichir cette didactisation en fournissant des contextes authentiques. On a une application indirecte du corpus dans le contexte de l'enseignement et de l'apprentissage de la langue et il est utilisé pour réajuster la qualité et la quantité d'activités et d'exercices. (Dechamps $2018: 358$ )

Nous choisissons tel ou tel type d'exercices et d'activités selon le temps disponible pour la préparation et la réalisation, les spécificités du document traité, l'intérêt et les compétences des étudiants, ainsi que leur besoin concret. On 
peut poser des questions oralement supposant des réponses quasi spontanées et assez courtes qui ont une dimension plutôt évaluative. Nous avons aussi l'habitude de donner aux étudiants plusieurs questions sous forme écrite, en leur donnant le temps pour réfléchir et chercher des réponses (en classe ou même parfois à la maison) et en respectant leur rythme individuel. Cette deuxième procédure demande à la totalité des étudiants un travail sur l'ensemble des questions (au lieu d'une participation active de chacun seulement à une ou deux questions dans la première procédure). Dans la première procédure la prise de parole est assez limitée, tandis que dans la deuxième, outre la compréhension, l'expression orale est également mise en œuvre, surtout pendant la concertation entre les étudiants.

En parlant des langues de spécialité on met l'accent sur l'importance du vocabulaire spécialisé et la compétence lexicale est vue non seulement comme nécessaire mais comme essentielle et principale parmi les compétences langagières utilisées dans la communication professionnelle. Heureusement, une grande partie du lexique universitaire et pédagogique comprend des termes internationaux d'origine latine ou grecque et donc déjà connus de nos étudiants. Des termes comme université, département, pédagogie, psychologie, système, instruction, orientation, autorité, autonomie, motivation etc. sont nombreux et facilitent donc la compréhension. Mais parmi eux il y a aussi des termes qui sont de faux-amis : ils sont immédiatement reconnaissables mais leur sens n'est pas le même dans toutes les langues, comme par exemple, méthodologie, méthode ou didactique. D'après notre expérience, leur explication et surtout traduction du français en serbe représentent une grande difficulté. (Vujovic $2018: 32$ )

Évidemment, le vocabulaire ne peut pas être réduit aux problèmes des mots isolés (il faut penser aux tournures syntaxiques spécifiques ainsi qu'à la dimension culturelle) ; cependant, la problématique du vocabulaire professionnel reste au premier plan. Toutefois, la question qui nous semble la plus importante reste ouverte : est-ce que toutes les notions pédagogiques, même celles qui sont essentielles et les plus fréquentes, sont bien claires et est-ce que nous connaissons leur traduction exacte dans la langue étrangère dont nous nous servons ? Nous savons tous qu'il existe de très grandes différences entre le vocabulaire pédagogique français et serbe. Nous avons essayé de les distinguer :

- Le lexique sans équivalent dans l'autre langue, ce qui est souvent la conséquence des différences culturelles entre les deux systèmes éducatifs (professeur des écoles, IUFM d'autrefois et ESPE d'aujourd'hui, collège, vanredni profesor, učiteljski fakultet, etc.)

- Les mots de la même origine qui se ressemblent beaucoup mais qui n'ont pas le même sens dans les deux langues (éducation / edukacija, obrazovanje, vaspitanje ; formation / formiranje, formacija, obrazovanje ; concours / konkurs, etc.) 
- Les mots de la même origine qui se ressemblent beaucoup mais qui n'ont pas le même emploi et la même extension du sens (école / škola ; classe / razred, odeljenje ; gymnase / gimnazija, didactique / didaktika, metodika, etc.)

- Les notions qui existent dans les deux langues et sont traduites facilement, mais qui n'ont pas le même sens: l'école primaire/élémentaire en France correspond uniquement au premier cycle de notre école primaire/ élémentaire ; l'enseignement primaire en France dure huit ans, comme en Serbie, mais répartis différemment (en France 3 ans de l'école maternelle +5 ans de l'école élémentaire, enfants de 3 à 11 ans; en Serbie deux cycles de 4 ans chacun, enfants entre 7 et 15 ans) ; l'enseignement secondaire en France dure sept ans (collège + lycée, enfants entre 11 et 18 ans) et en Serbie quatre ans (lycée, enfants entre 15 et 19 ans). (Vujovic $2018: 33$ )

Vus les problèmes et les confusions possibles, il serait préférable de faire un glossaire des notions universitaires et pédagogiques avec une présentation des systèmes scolaires de plusieurs pays francophones en comparaison avec ceux des pays serbophones. Nous pensons que ces informations seraient très utiles à tous les étudiants qui se préparent pour les divers métiers de l'enseignement et de l'éducation, ainsi que pour tous les enseignants actifs et les responsables de l'administration publique. Évidemment, il ne faut pas oublier qu'il y a toujours beaucoup de changements dans ce domaine, que les nouveaux sigles et les nouvelles appellations se multiplient et qu'il n'est jamais possible de les énumérer tous.

\section{En guise de conclusion}

Le FOU se fraie un chemin puisqu'il répond à un besoin urgent de développer chez les étudiants des compétences langagières, disciplinaires et méthodologiques nécessaires à la réussite de leurs études universitaires. Les initiatives se multiplient et traitent les besoins d'étudiants de nombreuses filières professionnelles, mais il serait tout de même très utile, voire nécessaire que les enseignants rassemblent leurs efforts et partagent leurs expériences (bonnes aussi bien que mauvaises) pour éviter toute perte du temps due aux répétitions des mêmes activités.

Il faut surtout prendre en considération plusieurs choses : s'adapter le plus possible aux besoins concrets de chaque groupe d'étudiants (selon le fait qu'ils partent pour un semestre entier ou pour un court stage pratique) ; insister sur un travail individuel sérieux et constant de chaque étudiant (vu que cette formation au FOU se fait souvent hors les cours prévus par le programme d'études suivi par tous nos étudiants francophones et qu'elle n'est destinée qu'à un nombre très 
restreint). Il ne faut surtout pas oublier l'essentiel - une forte motivation des étudiants qui exige de nombreux efforts de la part de l'enseignant : travailler beaucoup et établir un exemple personnel ; développer une bonne relation enseignant-apprenant et créer une atmosphère de travail agréable; augmenter l'autoconfiance linguistique des apprenants ; personnaliser le processus de l'apprentissage et promouvoir l'autonomie des étudiants. La motivation augmente si les étudiants deviennent conscients de leur propre parcours d'apprentissage et s'ils sont impliqués dans la création du programme.

\section{Références bibliographiques}

Courtillon 2003 : J. Courtillon, Elaborer un cours de FLE, Paris : Hachette.

Cuq, 2003 : J.P.Cuq (dir.), Dictionnaire du didactique du français langue étrangère et seconde, Paris : CLE International.

Mangiante \& Parpette 2004 : J.-M. Mangiante, Ch. Parpette, Le Français sur Objectif Spécifique : de l'analyse des besoins à l'élaboration d'un cours, Paris : Hachette.

Parpette 2018 : Ch. Parpette, « Le français sur objectif universitaire : entre contexte, savoir-faire didactique et stratégie institutionnelle », in A. Vujović, S. ŠipragićĐokić, M. Paprić (dir.), Langues sur objectifs spécifiques et identité professionnelle, Beograd: Društvo za strane jezike i književnosti Srbije, Učiteljski fakultet, 55-67.

Dechamps 2018 : C. Dechamps, « Corpus de spécialité et didactique du français sur objectif spécifique : quelles perspectives pour le français juridiques?» in A. Vujović, S. Šipragić-Đokić, M. Paprić (dir.), Langues sur objectifs spécifiques et identité professionnelle, Beograd: Društvo za strane jezike i književnosti Srbije, Učiteljski fakultet, 355-366.

Vujović 2018 : A. Vujović, « Notions de pédagogie en français et en serbe », Filolog, n 18, Banja Luka: Filološki fakultet Univerziteta u Banjoj Luci, 30-43. 
Ана Вујовић

\section{Француски језик за академске намене за учитеље и васпитаче}

За студенте нефилолошких факултета и стручњаке у свим областима познавање страних језика подразумева пре свега језичке компетенције у домену њихове (будуће) професије, што називамо страним језиком струке. Осим тога, последњих година интернационализација високог образовања и развој студентске мобилности у оквиру европских пројеката Еразмус+ у великој мери утичу на рад и организовање европских универзитета. Учитељски факултет Универзитета у Београду годинама развија успешну сарадњу са Високим школама за образовање наставника из Лиона и Бордоа. Наши студенти могу да на овим институцијама обаве део своје стручне праксе или да се укључе у програме прве и друге године мастер студија. Суочени са овим новим могућностима које се нуде нашим студентима, али и изазовима њихове адекватне језичке припреме за одлазак на поменуте француске високошколске установе, приступили смо осмишљавању и разрађивању посебног програма француског за академске намене намењеног управо будућим учитељима и васпитачима. Програм који овде представљамо плод је нашег вишегодишњег искуства у раду са овим студентима, али и размишљања представљених током семинара одржаног у марту 2018. на Учитељском факултету у Београду, а посвећеног француском језику струке и француском за академске намене. Полазнице семинара биле су универзитетске наставнице француског језика на филолошким и на нефилолошким факултетима, као и студенткиње француског језика на докторским и мастер студијама. На основу детаљне анализе конкретних потреба наших студената и компетенција које треба развијати, а користећи дескриптивну и компаративну методу, предлажемо неке могуће садржаје програма академског француског језика, наставне материјале и активности.

Кључне речи: француски језик за академске намене, компетенције, програм, наставни материјал, активности, учитељи и васпитачи. 